\title{
Stereodifferentiation in the Decay of Triplets and Biradicals Involved in \\ Intramolecular Hydrogen Transfer from Phenols or Indoles to $\pi, \pi *$ Aromatic
}

\section{Ketones}

Julia Pérez-Prieto, ${ }^{* a}$ Francisco Boscá, ${ }^{b}$ Agustín Lahoz, ${ }^{a}$ Martinez-Mañez, Ramón; ${ }^{b}$

$$
\text { Miguel A. Miranda*b }
$$

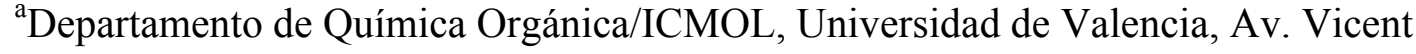
Andrés Estellés s/n, 46100 Burjassot, Valencia.

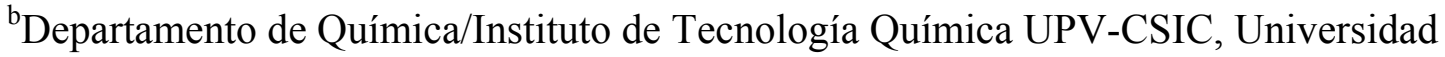
Politécnica de Valencia, Camino de Vera s/n, 46071 Valencia.

\section{Supplementary Material}

S2: Transient absorption spectra in deaerated metanol for (S,S)-3, (R,S)-3, (S,S)-5, (R,S)-1, (S,S)-1, (R,S)-2, (S,S)-2. S3: Triplet decay in metanol for (R,S)-3 and (S,S)-5. S4: Double reciprocal plots in metanol for $(\mathbf{R}, \mathbf{S})-\mathbf{1},(\mathbf{S}, \mathbf{S})-\mathbf{2},(\mathbf{R}, \mathbf{S})-\mathbf{3}$ and $(\mathbf{S}, \mathbf{S})-\mathbf{5}$. S5: Comparative decay of the biradicals in acetonitrile: (S,S)-7 and (R,S)-7, and (R,S)-9 and (S,S)-9. S6-S10: Characterization data for 1-6. S11-S17: Crystallographic data for (R,S)-2, (S,S)-3, (R,S)-5, (S,S)-5 

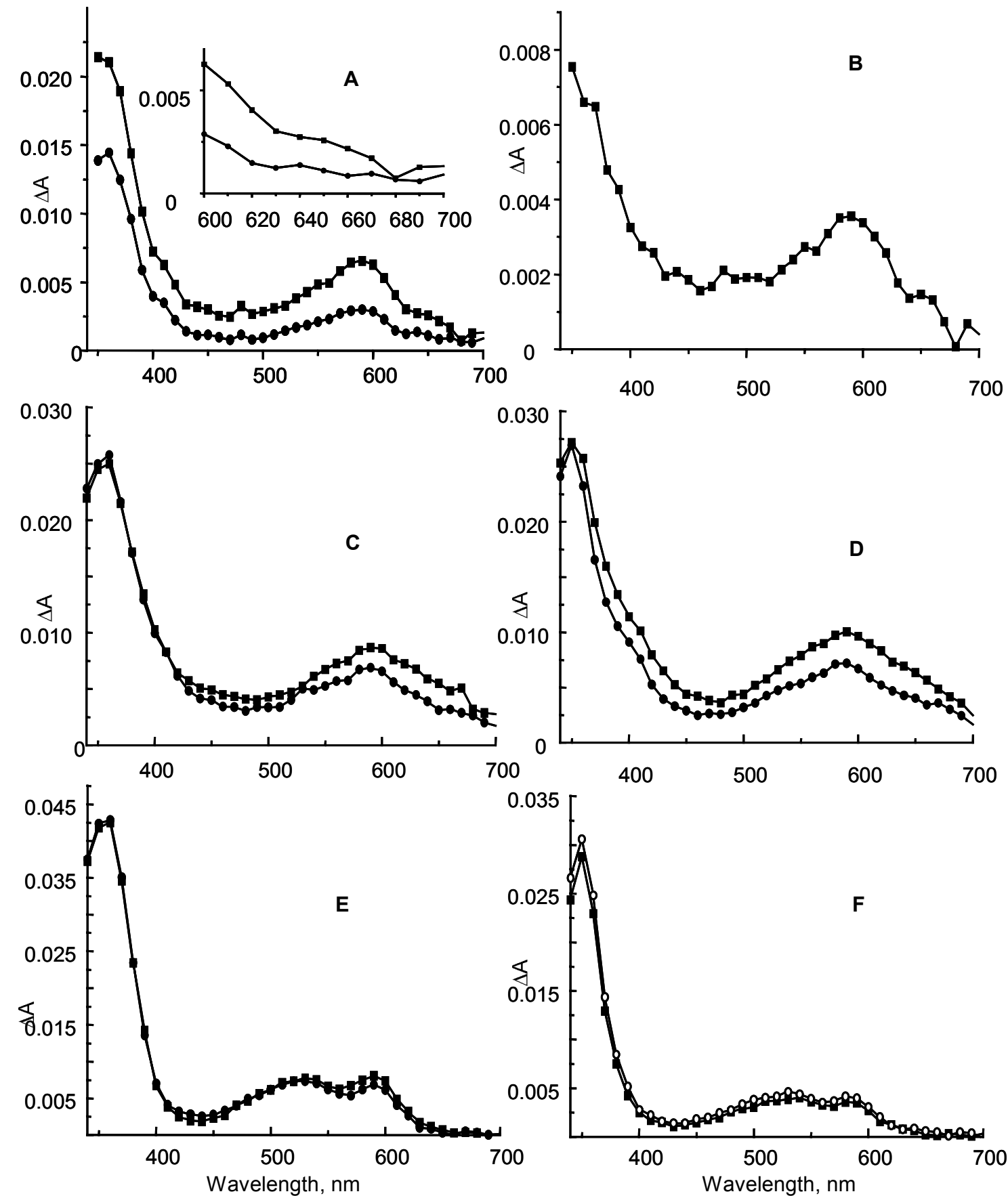

Figure 6. Transient absorption spectra recorded following laser excitation $(355 \mathrm{~nm})$ in deaerated methanol. A: of (S,S)-3, $10 \mathrm{~ns}(\bullet)$ and $50 \mathrm{~ns}(\bullet \quad)$ after the laser pulse. B: Difference spectrum of transient absorption spectra of $(S, S)-3$ recorded 10 ns and 50 ns after the laser pulse. C: of $(R, S)-3,15 \mathrm{~ns}(\mathrm{n})$ and $70 \mathrm{~ns}(\bullet)$ ) after the laser pulse. D:of $(S, S)-5,15 \mathrm{~ns}($. ) and $70 \mathrm{~ns}(\bullet)$ after the laser pulse.E:of $(R, S)-1,30 \mathrm{~ns}(\mathbf{})$ and $(S, S)-1,15 \mathrm{~ns}(\bullet)$ after the laser pulse. F:of $(R, S)-2,30 \mathrm{~ns}($.$) and (S, S)-2,30 \mathrm{~ns}$ (o) after the laser pulse. 

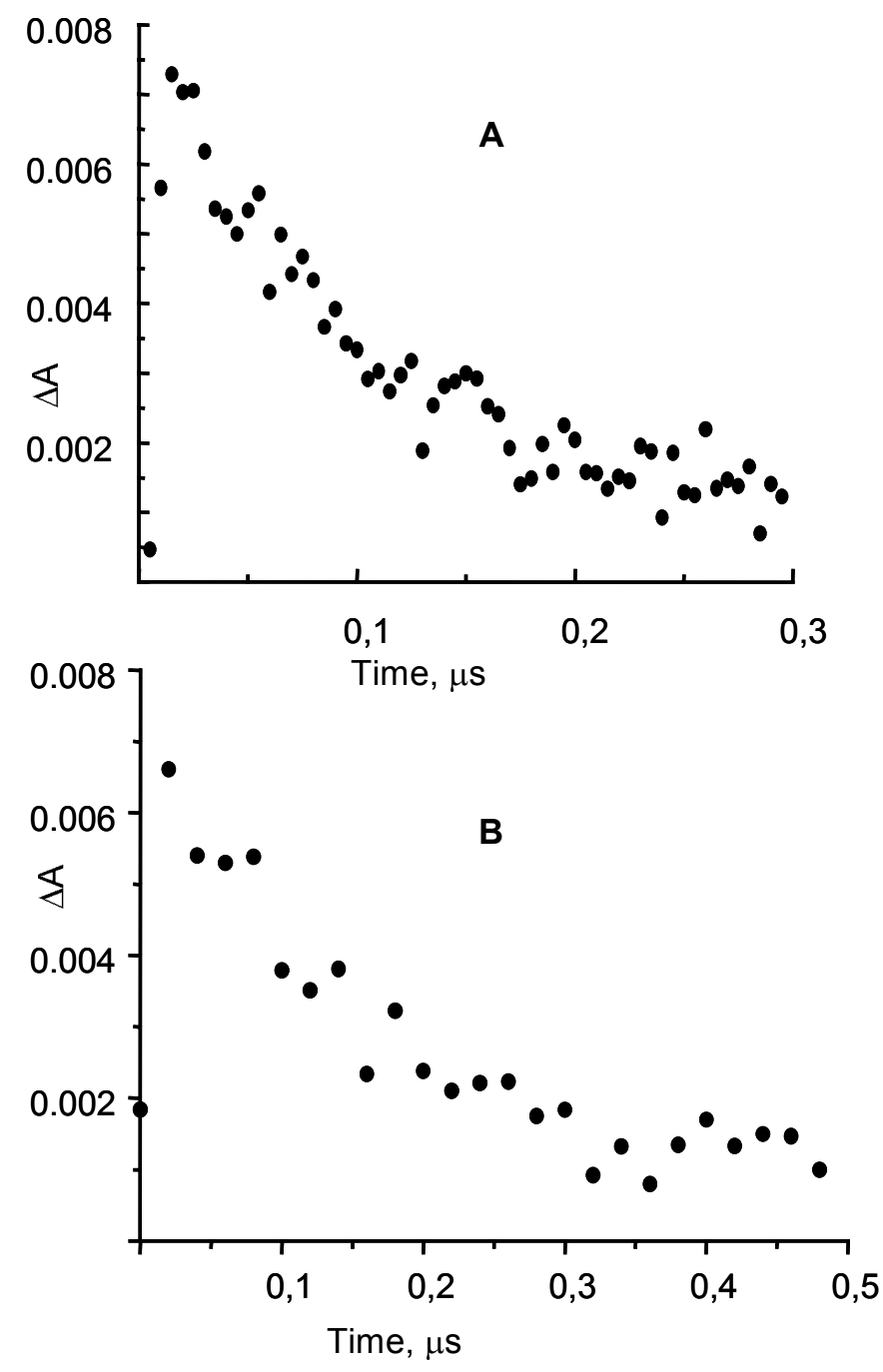

Figure 7. Triplet decay monitored at $630 \mathrm{~nm}$, following laser excitation (355 nm) in deaerated methanol: A: for $(R, S)-3$ and B: for $(S, S)-5$. 


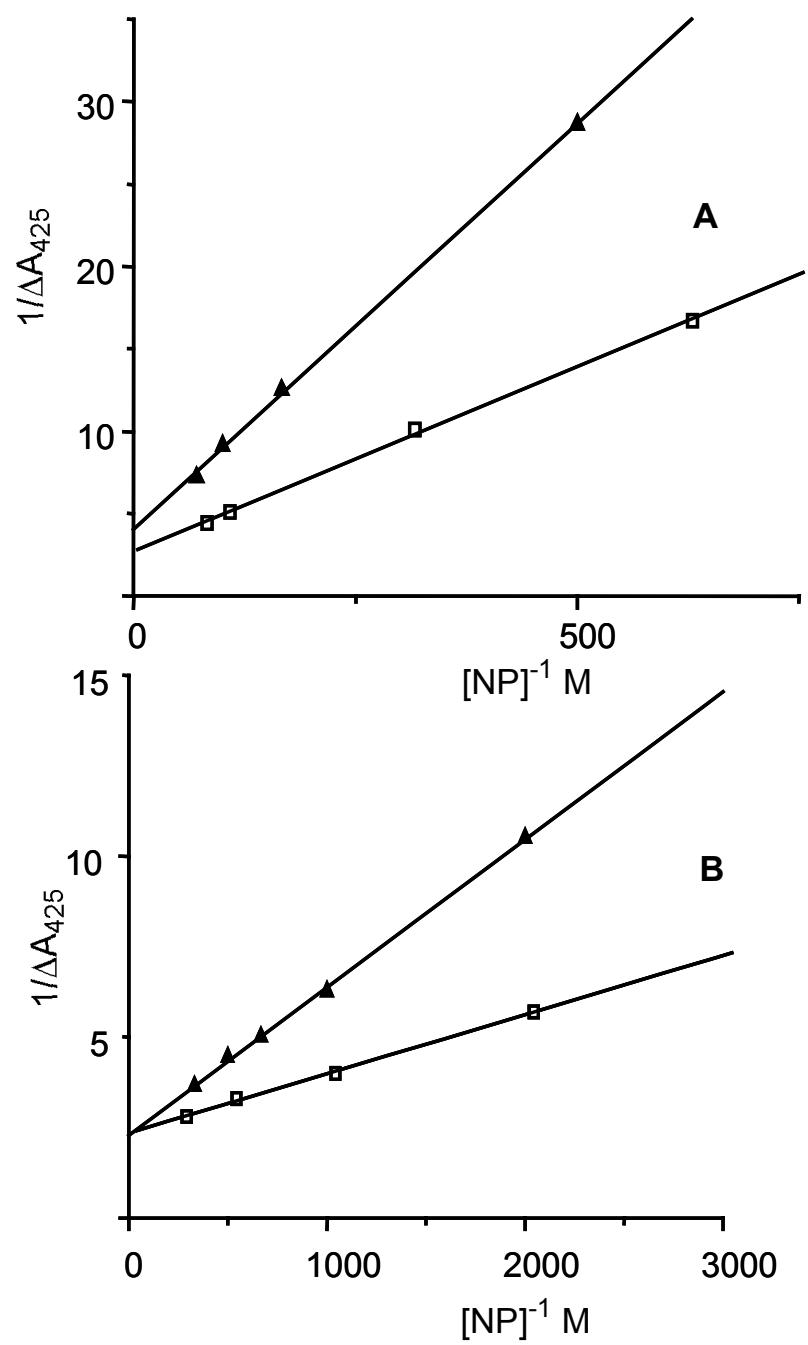

Figure 8. A: Double reciprocal plots for quenching of the ketone triplets generated by laser flash photolysis at $355 \mathrm{~nm}$ in deaerated methanolic solutions of A: $(R, S)-1(\Lambda)$ and $(S, S)-2(\square)$, and B: of $(R, S)-3(\Lambda)$ and $(S, S)-5(\square)$. 

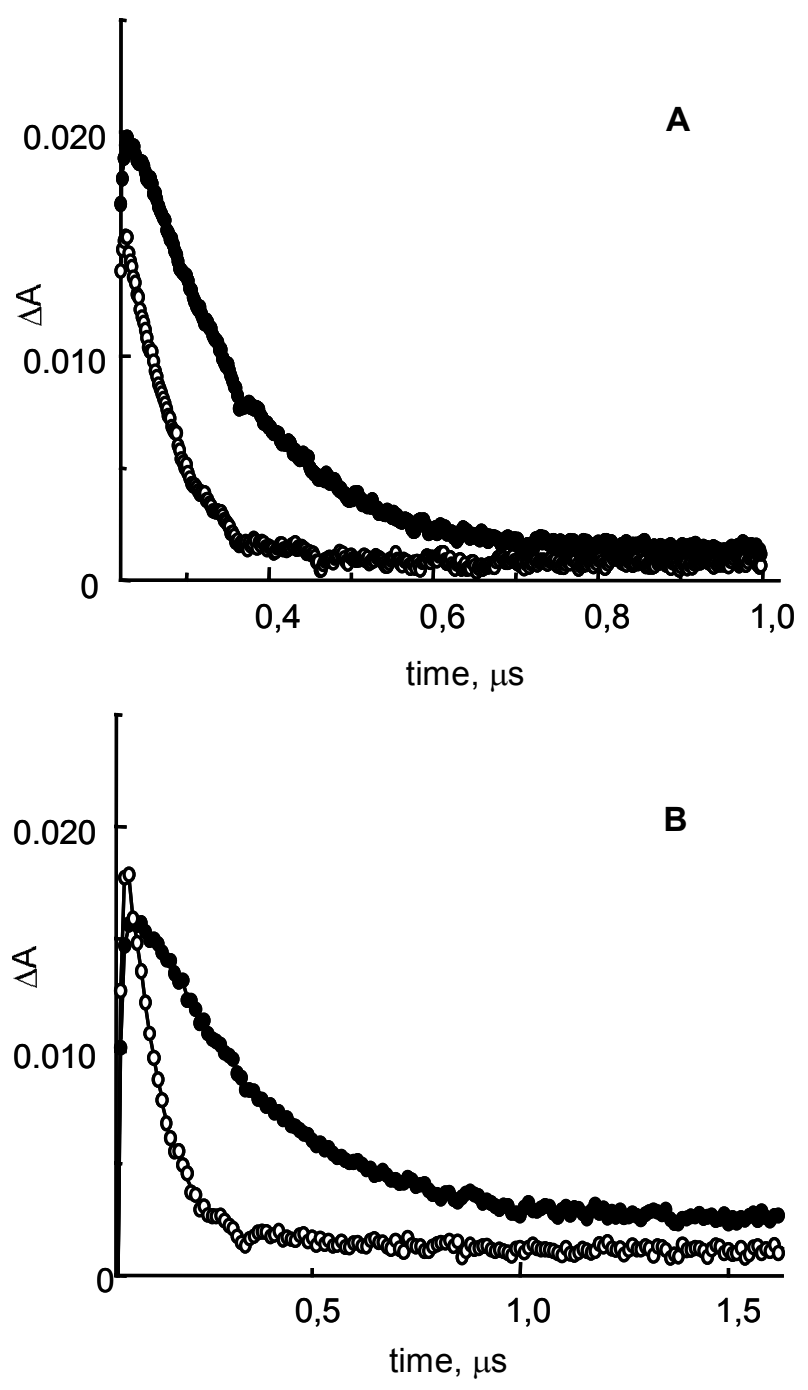

Figure 9. Comparative decay of the biradicals of diastereoisomers monitored at $360 \mathrm{~nm}$, following laser excitation $(355 \mathrm{~nm})$ in deaerated acetonitrile: A: $(S, S)-7(0)$ and $(R, S)-7(\bullet)$, B: $(R, S)-9(\bullet)$ and $(S, S)-9(\circ)$. 
Chemicals. 2-(5-Benzoylthien-2-yl)propanoic acid (tiaprofenic acid, TPA) was extracted from commercial samples using dichloromethane as solvent. 2-[4-(thien-2ylcarbonyl)phenyl]propanoic acid (suprofen, SP), (L)-tyrosine methyl ester, (L)tryptophan methyl ester, [1-ethyl-3-(3-(dimethylamino)propylcarbodiimide] (EDC), 1hydroxybenzotriazole (HBT), were commercially available.

General Procedure. All synthetic procedures were performed in a dry nitrogen atmosphere using purified dried solvents. ${ }^{1} \mathrm{H}-\mathrm{NMR}$ spectra were recorded in a $300 \mathrm{MHz}$ spectrometer, chemical shifts $(\delta)$ are reported in ppm relative to TMS. The coupling constants $(J)$ are in hertz $(\mathrm{Hz})$. Column chromatography was performed on silica gel (230-400 mesh). Eluent composition is given as volume/volume mixtures unless specified otherwise. HPLC was carried out using a C-18 column or a Silica column.

Synthesis of bichromophores 1-3 and 5. The corresponding propanoic acid (300 $\mathrm{mg}, 1.2 \mathrm{mmol})$, the $(\boldsymbol{S})$-amino acid methyl ester (1.4 mmol), EDC (270 $\mathrm{mg}, 1.4$ mmol) and HBT (200 mg, $1.4 \mathrm{mmol})$ were combined in dry, degassed DMF (10 mL) at room temperature. The $\mathrm{pH}$ was adjusted to 9.0-9.5 with triethylamine and the mixture was stirred overnight. Afterwards, the solvent was removed in vacuo, and the residue was treated with $10 \% \mathrm{HCl}(30 \mathrm{~mL})$ and extracted with ethyl acetate $(3 \times 10 \mathrm{~mL})$. The combined organic layers were washed with $10 \% \mathrm{HCl}(1 \times 10 \mathrm{~mL})$, saturated sodium bicarbonate $(2 \times 10 \mathrm{~mL})$ and brine $(1 \times 10 \mathrm{~mL})$ and dried over sodium sulfate. Solvent was removed under reduced pressure to give a yellow oil. The residue was chromatographed (hexane/ethyl acetate, 2:1) leading to the corresponding bichromophore as a 1:1 diasteroisomeric mixture. Separation of diasteroisomers was accomplished by HPLC, mobile phase: potassium dihydrogen phosphate (0.06 M): acetonitrile: triethylamine (317: 183: 0.1$)$ flow rate $1.5 \mathrm{~mL} \min ^{-1}$; UV detection $\lambda=310$ $\mathrm{nm}$. 
O-Methylation of the tyrosine-derived bichromophores. An ethereal solution containing compounds $\mathbf{3}$ or $\mathbf{5}$, as diastereoisomeric mixture, (300 mg, $0.7 \mathrm{mmol})$, was treated with $20 \mathrm{mmol}$ of freshly prepared diazomethane ${ }^{16}$ at $0^{\circ} \mathrm{C}$. The mixture was allowed to warm up to room temperature and stirred overnight. Afterwards, the excess of diazomethane was removed under reduced pressure and the resulting yellow oil was chromatographed (hexane/ethyl acetate, 3:1) leading to the corresponding bichromophore as a 1:1 diasteromeric mixture. Separation of the diasteroisomers was accomplished by HPLC: stationary phase Silica column; mobile phase: hexane: ethyl acetate ( $4: 1$ by volume) flow rate $2 \mathrm{~mL} \mathrm{~min}^{-1}$; detection by refractive index.

Compounds 1: Yield: $54 \%$

Methyl N-[(2S)-2-(5-benzoylthien-2-yl)propanoyl-3-(1H-indol-6-yl)-(S)alaninate $[(S, S)-1]$.

${ }^{1} \mathrm{H}-\mathrm{NMR}\left(\mathrm{CDCl}_{3}\right): 1.46(\mathrm{~d}, J=7.1 \mathrm{~Hz}, 3 \mathrm{H}), 3.18(\mathrm{~d}, J=5.5 \mathrm{~Hz}, 2 \mathrm{H}), 3.62(\mathrm{~s}$, $3 \mathrm{H}), 3.72(\mathrm{q}, J=7.1 \mathrm{~Hz}, 1 \mathrm{H}), 4.83(\mathrm{~m}, 1 \mathrm{H}), 6.16(\mathrm{~d}, J=7.8 \mathrm{~Hz}, 1 \mathrm{H}), 6.69(\mathrm{~s}, 1 \mathrm{H}), 6.78$ $(\mathrm{d}, J=3.9 \mathrm{~Hz}, 1 \mathrm{H}), 6.98(\mathrm{t}, J=6.9 \mathrm{~Hz}, 1 \mathrm{H}), 7.04(\mathrm{t}, J=6.9 \mathrm{~Hz}, 1 \mathrm{H}), 7.20(\mathrm{~d}, J=7.1$ $\mathrm{Hz}, 1 \mathrm{H}), 7.33-7.60(\mathrm{~m}, 5 \mathrm{H}), 7.75(\mathrm{~d}, J=7.7 \mathrm{~Hz}, 2 \mathrm{H}), 8.4(\mathrm{~s}, 1 \mathrm{H}) .{ }^{13} \mathrm{C}-\mathrm{NMR}\left(\mathrm{CDCl}_{3}\right): \delta$ 19.4, 27.3, 29.6, 42.7, 52.4, 52.9. 109.2, 111.3, 118.3, 119.5, 122.1, 123.1, 126.1, 127.3, $128.4,129.1,132.3,135.1,136.1,137.8,141.9,153.3,171.6,172.2,188.2 . \quad \mathrm{MS} \mathrm{m} / z$ $460\left(\mathrm{M}^{+}\right.$6), 201 (53), 130 (100). Anal. Calcd for $\mathrm{C}_{26} \mathrm{H}_{24} \mathrm{~N}_{2} \mathrm{O}_{4} \mathrm{~S}: \mathrm{C}, 67.81 ; \mathrm{H}, 5.25$. Found: C, 67.17; H, 5.33. HRMS Calcd for $\mathrm{C}_{26} \mathrm{H}_{24} \mathrm{~N}_{2} \mathrm{OS}_{4}$ : 460.1456. Found: 460.1451.

Methyl N-l(2S)-2-(5-benzoylthien-2-yl)propanoyl-3-(1H-indol-6-yl)-(S)-alaninate [(R, S)-1]. ${ }^{1} \mathrm{H}-\mathrm{NMR}\left(\mathrm{CDCl}_{3}\right): 1.47(\mathrm{~d}, J=7.1 \mathrm{~Hz}, 3 \mathrm{H}), 3.22(\mathrm{~m}, 2 \mathrm{H}), 3.60(\mathrm{~s}, 3 \mathrm{H}), 3.72$ $(\mathrm{q}, J=7.1 \mathrm{~Hz}, 1 \mathrm{H}), 4.79(\mathrm{~m}, 1 \mathrm{H}), 6.16(\mathrm{~d}, J=7.7 \mathrm{~Hz}, 1 \mathrm{H}), 6.79(\mathrm{~m}, 2 \mathrm{H}), 7.00(\mathrm{t}, J=6.9$ $\mathrm{Hz}, 1 \mathrm{H}), 7.07(\mathrm{t}, J=7.0 \mathrm{~Hz}, 1 \mathrm{H}), 7.23(\mathrm{~d}, J=7.5 \mathrm{~Hz}, 1 \mathrm{H}), 7.32-7.49(\mathrm{~m}, \mathrm{~d}, 5 \mathrm{H}), 7.74$ 
$(\mathrm{d}, J=6.9 \mathrm{~Hz}, 2 \mathrm{H}), 8.44(\mathrm{~s}, 1 \mathrm{H}) .{ }^{13} \mathrm{C}-\mathrm{NMR}\left(\mathrm{CDCl}_{3}\right): \delta$ 19.3, 27.2, 29.6, 42.9, 52.4, 53.0. 109.3, 111.4, 118.3, 119.5, 122.1, 122.9, 126.1, 127.4, 128.4, 129.1, 132.3, 135.1, 136.1, 137.8, 142.0, 153.1, 171.7, 172.2, 189.0. $\mathrm{MS} \mathrm{m} / z \quad 460\left(\mathrm{M}^{+} 6\right), 201(70), 130$ (100). HRMS Calcd for $\mathrm{C}_{26} \mathrm{H}_{24} \mathrm{~N}_{2} \mathrm{O}_{4} \mathrm{~S}: 460.1456$. Found: 460.1454.

Compounds 2: Yield: $51 \%$

Methyl (1H-indol-6-yl)-N-\{(2S)-2-[4-(thien-2-ylcarbonyl)phenyl]propanoyl\}(S)-alaninate [(S, S)-2]. ${ }^{1} \mathrm{H}-\mathrm{NMR}\left(\mathrm{CDCl}_{3}\right): 1.47(\mathrm{~d}, J=7.1 \mathrm{~Hz}, 3 \mathrm{H}), 3.15(\mathrm{~m}, 2 \mathrm{H}), 3.54$ $(\mathrm{q}, J=7.1 \mathrm{~Hz}, 1 \mathrm{H}), 3.59(\mathrm{~s}, 3 \mathrm{H}), 4.70\left(\mathrm{dd}, J_{1}=4.5 \mathrm{~Hz}, J_{2}=2.75 \mathrm{~Hz}, 1 \mathrm{H}\right), 5.72(\mathrm{~d}, J=$ $6.5 \mathrm{~Hz}, 1 \mathrm{H}), 6.42(\mathrm{~s}, 1 \mathrm{H}), 6.99-7.8(\mathrm{~m}, 11 \mathrm{H}), 8.47(\mathrm{~s}, 1 \mathrm{H}) .{ }^{13} \mathrm{C}-\mathrm{NMR}\left(\mathrm{CDCl}_{3}\right): \delta 17.9$, $27.1,47.0,52.3,52.4,109.1,111.4,118.4,119.5,122.2,122.7,127.8,128.2,129.5$, 134.8, 135.3, 136.1, 137.1, 143.3, 145.1, 172.1, 173.1, 188.6. MS $m / z 460\left(\mathrm{M}^{+} 6\right), 201$ (62), 130 (100). HRMS Calcd for $\mathrm{C}_{26} \mathrm{H}_{24} \mathrm{~N}_{2} \mathrm{O}_{4} \mathrm{~S}$ : 460.1456. Found: 460.1471.

Methyl (1H-indol-6-yl)-N-\{(2R)-2-[4-(thien-2-ylcarbonyl)phenyl]propanoyl\}(S)-alaninate [(R, S)-2].

${ }^{1} \mathrm{H}-\mathrm{NMR}\left(\mathrm{CDCl}_{3}\right): 1.45(\mathrm{~d}, J=7.2 \mathrm{~Hz}, 3 \mathrm{H}), 3.10(\mathrm{~m}, 2 \mathrm{H}), 3.58(\mathrm{q}, J=7.2 \mathrm{~Hz}$, 1H), $3.62(\mathrm{~s}, 3 \mathrm{H}), 4.71(\mathrm{~m}, 1 \mathrm{H}), 5.76(\mathrm{~d}, J=7.8 \mathrm{~Hz}, 1 \mathrm{H}), 6.20(\mathrm{~s}, 1 \mathrm{H}), 6.90-7.40(\mathrm{~m}, 7 \mathrm{H})$, 7.50-7.70 (m, 4H), 8.47 (s, 1H). MS m/z $460\left(\mathrm{M}^{+} 4\right), 201$ (83), 130 (100). HRMS Calcd for $\mathrm{C}_{26} \mathrm{H}_{24} \mathrm{~N}_{2} \mathrm{O}_{4} \mathrm{~S}: 460.1456$. Found: 460.1457 .

Compounds 3: Yield: 55\%

Methyl N-[(2S)-2-(5-benzoylthien-2-yl)propanoyl]-(S)-tyrosinate [(S,S)-3]. ${ }^{1} \mathrm{H}-$ $\operatorname{NMR}\left(\mathrm{CDCl}_{3}\right): 1.53(\mathrm{~d}, J=7.0 \mathrm{~Hz}, 3 \mathrm{H}), 2.86\left(\mathrm{dd}, J_{1}=14 \mathrm{~Hz}, J_{2}=5 \mathrm{~Hz}, 1 \mathrm{H}\right), 3.00(\mathrm{dd}$, $\left.J_{1}=14 \mathrm{~Hz}, J_{2}=5.0 \mathrm{~Hz}, 1 \mathrm{H}\right), 3.69(\mathrm{~s}, 3 \mathrm{H}), 3.77(\mathrm{q}, J=7.0 \mathrm{~Hz}, 1 \mathrm{H}), 4.75(\mathrm{~m}, 1 \mathrm{H}) ; 5.85$ $(\mathrm{d}, J=7.2 \mathrm{~Hz}, 1 \mathrm{H}), 6.57(\mathrm{~d}, J=8.6 \mathrm{~Hz}, 2 \mathrm{H}), 6.68(\mathrm{~d}, J=8.6 \mathrm{~Hz}, 2 \mathrm{H}), 6.88(\mathrm{~d}, J=4.0$ $\mathrm{Hz}, 1 \mathrm{H}) ; 7.45(\mathrm{~m}, 5 \mathrm{H}), 7.81(\mathrm{~d}, J=8.4 \mathrm{~Hz}, 2 \mathrm{H})$. Anal. Calcd for $\mathrm{C}_{24} \mathrm{H}_{23} \mathrm{NO}_{5} \mathrm{~S}: \mathrm{C}, 65.89$; H, 5.30. Found: C, 65.86; H, 5.42. 
Methyl N-I(2R)-2-(5-benzoylthien-2-yl)propanoyl]-(S)-tyrosinate [(R,S)-3]. ${ }^{1} \mathrm{H}-$ $\operatorname{NMR}\left(\mathrm{CDCl}_{3}\right): 1.49(\mathrm{~d}, J=7.0 \mathrm{~Hz}, 3 \mathrm{H}), 2.88\left(\mathrm{dd}, J_{1}=14.0 \mathrm{~Hz}, J_{2}=6.2 \mathrm{~Hz}, 1 \mathrm{H}\right), 3.00$ $\left(\mathrm{dd}, J_{1}=14.0 \mathrm{~Hz}, J_{2}=6.2 \mathrm{~Hz}, 1 \mathrm{H}\right), 3.64(\mathrm{~s}, 3 \mathrm{H}), 3.82(\mathrm{q}, J=7.0 \mathrm{~Hz}, 1 \mathrm{H}), 4.72(\mathrm{~m}, 1 \mathrm{H})$, $6.23(\mathrm{~d}, J=7.9 \mathrm{~Hz}, 1 \mathrm{H}), 6.63(\mathrm{~d}, J=8.3 \mathrm{~Hz}, 2 \mathrm{H}), 6.77(\mathrm{~d}, J=8.3 \mathrm{~Hz}, 2 \mathrm{H}), 6.89(\mathrm{~d}, J=$ $3.9 \mathrm{~Hz}, 1 \mathrm{H}), 7.30-7.51(\mathrm{~m}, 5 \mathrm{H}), 7.74(\mathrm{~d}, J=7.2 \mathrm{~Hz}, 2 \mathrm{H}) .{ }^{13} \mathrm{C}-\mathrm{NMR}\left(\mathrm{CDCl}_{3}\right): \delta 19.4$, $36.7,42.8,52.4,53.3,115.6,126.2,126.6,128.4,129.1,130.2,132.4,135.4,137.7$, 142.1, 153.0, 155.5, 171.9, 172.0, 188.3. MS m/z $438\left(\mathrm{M}^{+} 60\right), 436$ (100), 404 (16), 260 (36), 189 (85), 107 (55). HRMS Calcd for $\mathrm{C}_{24} \mathrm{H}_{23} \mathrm{NO}_{5} \mathrm{~S}: 438.1375$. Found: 438.1355.

Compounds 4: Yield: 57\%

Methyl N-I(2R)-2-(5-benzoylthien-2-yl)propanoyl]-O-methyl-(S)-tyrosinate [R,S)-4]. ${ }^{1} \mathrm{H}-\mathrm{NMR}\left(\mathrm{CDCl}_{3}\right): 1.52(\mathrm{~d}, J=7.0 \mathrm{~Hz}, 3 \mathrm{H}), 3.02(\mathrm{~m}, 2 \mathrm{H}), 3.66(\mathrm{~s}, 3 \mathrm{H}), 3.67$ $(\mathrm{s}, 3 \mathrm{H}), 3.81(\mathrm{q}, J=7.0 \mathrm{~Hz}, 1 \mathrm{H}), 4.78(\mathrm{~m}, 1 \mathrm{H}), 5.92(\mathrm{~d}, J=7.8 \mathrm{~Hz}, 1 \mathrm{H}), 6.70(\mathrm{~d}, J=8.4$ $\mathrm{Hz}, 2 \mathrm{H}), 6.84(\mathrm{~d}, J=8.4 \mathrm{~Hz}, 2 \mathrm{H}), 6.91(\mathrm{~d}, J=3.5 \mathrm{~Hz}, 1 \mathrm{H}), 7.4-7.5(\mathrm{~m}, 4 \mathrm{H}), 7.52(\mathrm{t}, J=$ $7.5 \mathrm{~Hz}, 1 \mathrm{H}), 7.78$ (d, $J=7.8 \mathrm{~Hz}, 2 \mathrm{H}) . \mathrm{MS} \mathrm{m} / z 451\left(\mathrm{M}^{+} 4\right), 216$ (13), 215 (14), 192 (100). HRMS Calcd for $\mathrm{C}_{25} \mathrm{H}_{25} \mathrm{NO}_{5} \mathrm{~S}: 451.1453$. Found: 451.1466.

Methyl N-[(2S)-2-(5-benzoylthien-2-yl)propanoyl]-O-methyl-(S)-tyrosinate [S,S)-4]. ${ }^{1} \mathrm{H}-\mathrm{NMR}\left(\mathrm{CDCl}_{3}\right): 1.53(\mathrm{~d}, J=7.0 \mathrm{~Hz}, 3 \mathrm{H}), 2.97(\mathrm{~m}, 2 \mathrm{H}), 3.64(\mathrm{~s}, 3 \mathrm{H}), 3.68$ (s, 3H), 3.79 (q, $J=7.0 \mathrm{~Hz}, 1 \mathrm{H}), 4.78(\mathrm{~m}, 1 \mathrm{H}), 5.90(\mathrm{~d}, J=7.5 \mathrm{~Hz}, 1 \mathrm{H}), 6.64(\mathrm{~d}, J=$ $8.4 \mathrm{~Hz}, 2 \mathrm{H}), 6.74(\mathrm{~d}, J=8.4 \mathrm{~Hz}, 2 \mathrm{H}), 6.88(\mathrm{~d}, J=3.6 \mathrm{~Hz}, 1 \mathrm{H}), 7.4-7.6(\mathrm{~m}, 5 \mathrm{H}), 7.79(\mathrm{~d}$, $J=7.8 \mathrm{~Hz}, 2 \mathrm{H})$. Anal. Calcd for $\mathrm{C}_{25} \mathrm{H}_{25} \mathrm{NO}_{5} \mathrm{~S}: \mathrm{C}, 66.50 ; \mathrm{H}, 5.58$. Found: C, 66.23; H, 5.51 .

Compounds 5: Yield: $61 \%$

Methyl N-\{(2S)-2-[4-thien-2-ylcarbonyl)phenyl]propanoyl\}-(S)-

tyrosinate $[(\boldsymbol{R}, S)-5] .{ }^{1} \mathrm{H}-\mathrm{NMR}: 1.50(\mathrm{~d}, J=7.0 \mathrm{~Hz}, 3 \mathrm{H}), 2.75\left(\mathrm{dd}, J_{1}=14 \mathrm{~Hz}, J_{2}=8 \mathrm{~Hz}\right.$, 
$1 \mathrm{H}), 3.10\left(\mathrm{dd}, J_{1}=14 \mathrm{~Hz}, J_{2}=8 \mathrm{~Hz}, 1 \mathrm{H}\right), 3.60(\mathrm{q}, J=7.0 \mathrm{~Hz}, 1 \mathrm{H}), 3.80(\mathrm{~s}, 3 \mathrm{H}), 4.75$ (m, 1H), 5.60 (d, J=8.2 Hz, 1H), 6.45-6.60 (m, 5H), 7.20 (m, 2H), 7.69-7.80 (m, 4H).

Methyl N-\{(2S)-2-[4-thien-2-ylcarbonyl)phenyl]propanoyl\}-(S)-tyrosinate [(S,S)-5]. ${ }^{1} \mathrm{H}-\mathrm{NMR}: 1.55(\mathrm{~d}, J=7.2 \mathrm{~Hz}, 3 \mathrm{H}), 2.95(\mathrm{~m}, 2 \mathrm{H}), 3.70(\mathrm{~m}, 4 \mathrm{H}), 4.69$ (m, 1H), $5.80(\mathrm{~d}, J=7.6 \mathrm{~Hz}, 1 \mathrm{H}), 6.60(\mathrm{~d}, J=8.6 \mathrm{~Hz}, 2 \mathrm{H}), 6.70(\mathrm{~d}, J=8.6 \mathrm{~Hz}, 2 \mathrm{H}), 7.18-7.35$ (m, 4H), 7.55 (m, 1H), $7.65(\mathrm{~m}, 3 \mathrm{H})$. Anal. Calcd for $\mathrm{C}_{24} \mathrm{H}_{23} \mathrm{NO}_{5} \mathrm{~S}: \mathrm{C}, 65.89$; H, 5.30; N, $3.20 ;$ S, 7.33. Found : C, 65.83; H, 5.44; N, 3.24 ; S, 7.14.

Compounds 6. Yield: $57 \%$

Methyl O-methyl-N-\{(2S)-2-[4-thien-2-ylcarbonyl)phenyl]propanoyl\}-(S)tyrosinate $[(\boldsymbol{S}, \boldsymbol{S})-6] \cdot{ }^{1} \mathrm{H}-\mathrm{NMR}\left(\mathrm{CDCl}_{3}\right): 1.53(\mathrm{~d}, J=7.0 \mathrm{~Hz}, 3 \mathrm{H}), 3.01(\mathrm{~m}, 2 \mathrm{H}), 3.62(\mathrm{q}$, $J=7.0 \mathrm{~Hz}, 1 \mathrm{H}), 3.72(\mathrm{~s}, 3 \mathrm{H}), 3.75(\mathrm{~s}, 3 \mathrm{H}), 4.86(\mathrm{~m}, 1 \mathrm{H}), 5.82(\mathrm{~d}, J=7.8 \mathrm{~Hz}, 1 \mathrm{H}), 6.65$ $(\mathrm{s}, 4 \mathrm{H}), 7.20\left(\mathrm{dd}, J_{1}=4.9 \mathrm{~Hz}, J_{2}=3.8 \mathrm{~Hz}, 1 \mathrm{H}\right), 7.38(\mathrm{~d}, J=8.2 \mathrm{~Hz}, 2 \mathrm{H}), 7.67(\mathrm{~d}, J=3.8$ Hz, 1H), $7.75(\mathrm{~d}, J=4.8 \mathrm{~Hz}, 1 \mathrm{H}), 7.86(\mathrm{~d}, J=8.2 \mathrm{~Hz}, 2 \mathrm{H}) . \mathrm{MS} m / z 451\left(\mathrm{M}^{+} 2\right), 392$ (2), 192 (100), 121 (59). HRMS Calcd for $\mathrm{C}_{25} \mathrm{H}_{25} \mathrm{NO}_{5} \mathrm{~S}$ : 451.1453. Found: 451.1455.

\section{Methyl O-methyl-N-\{(2R)-2-[4-thien-2-ylcarbonyl)phenyl]propanoyl\}-(S)-tyrosinate} [(R,S)-6]. ( $\boldsymbol{R}, \boldsymbol{S})$-SUP-TyrOMe. ${ }^{1} \mathrm{H}-\mathrm{NMR}\left(\mathrm{CDCl}_{3}\right): 1.52$ (d, $\left.J=7.0 \mathrm{~Hz}, 3 \mathrm{H}\right), 2.99$ (dd, $\left.J_{1}=11.7 \mathrm{~Hz}, J_{2}=4.8 \mathrm{~Hz}, 1 \mathrm{H}\right), 3.08\left(\mathrm{dd}, J_{1}=14.0 \mathrm{~Hz}, J_{2}=4.8 \mathrm{~Hz}, 1 \mathrm{H}\right), 3.65(\mathrm{q}, J=7.0$ $\mathrm{Hz}, 1 \mathrm{H}), 3.70(\mathrm{~s}, 3 \mathrm{H}), 3.75(\mathrm{~s}, 3 \mathrm{H}), 4.78(\mathrm{~m}, 1 \mathrm{H}), 5.88(\mathrm{~d}, J=7.6 \mathrm{~Hz}, 1 \mathrm{H}), 6.77(\mathrm{~d}, J=$ $8.7 \mathrm{~Hz}, 2 \mathrm{H}), 6.87(\mathrm{~d}, J=8.7 \mathrm{~Hz}, 2 \mathrm{H}), 7.17\left(\mathrm{dd}, J_{1}=4.9 \mathrm{~Hz}, J_{2}=3.7 \mathrm{~Hz}, 1 \mathrm{H}\right), 7.40(\mathrm{~d}, J$ $=8.2 \mathrm{~Hz}, 2 \mathrm{H}), 7.64(\mathrm{~d}, J=3.7 \mathrm{~Hz}, 1 \mathrm{H}), 7.73(\mathrm{~d}, J=4.8 \mathrm{~Hz}, 1 \mathrm{H}), 7.83(\mathrm{~d}, J=8.2 \mathrm{~Hz}$, 2H). MS m/z $451\left(\mathrm{M}^{+} 2\right), 392$ (3), 192 (100), 121 (57). HRMS Calcd for $\mathrm{C}_{25} \mathrm{H}_{25} \mathrm{NO}_{5} \mathrm{~S}$ : 451.1453. Found: 451.1440. 


\section{Crystallographic Data}

Compound $(\boldsymbol{S}, \boldsymbol{S})-3 . \mathrm{C}_{23} \mathrm{H}_{21} \mathrm{~N}_{5} \mathrm{~S}, M=423.47$, triclinic space group $P 1, a=7.889(5) \AA$, $b=8.294(5) \AA, c=8.727(5) \AA, \alpha=79.70(5)^{\circ}, \beta=74.71(4)^{\circ}, \gamma=87.15(5)^{\circ}, V=$

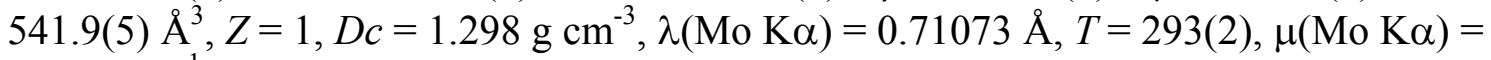
$0.183 \mathrm{~mm}^{-1}$. A well-shaped crystal of dimensions $0.18 \times 0.18 \times 0.22 \mathrm{~mm}$ was mounted on a Siemens P4 diffractometer. A total of 8553 independent reflections were collected. The structure was solved by direct methods and refined by full-matrix least-squares analysis on $F^{2}$ (SHELXTL). The refinement converged to $R_{1}=0.029[F>4 \sigma(F)], w R_{2}$ $=0.087$ (all data) and $G O F=0.815$. Absolute structure parameter $=0.03(6)$.

Compound (R,S)-5. $\mathrm{C}_{24} \mathrm{H}_{23} \mathrm{N0}_{5} \mathrm{~S}, M=437.49$, triclinic space group $P 1, a=7.902(2) \AA$, $b=8.256(3) \AA, c=8.859(3) \AA, \alpha=78.40(2)^{\circ}, \beta=76.73(2)^{\circ}, \gamma=87.96(3)^{\circ}, V=$ $551.0(3) \AA^{3}, Z=1, D c=1.318 \mathrm{~g} \mathrm{~cm}^{-3}, \lambda($ Mo K $\alpha)=0.71073 \AA, T=293(2), \mu($ Mo K $\alpha)=$ $0.182 \mathrm{~mm}^{-1}$. A well-shaped crystal of dimensions $0.18 \times 0.20 \times 0.20 \mathrm{~mm}$ was mounted on a Siemens P4 diffractometer. A total of 8553 independent reflections were collected. The structure was solved by direct methods and refined by full-matrix least-squares analysis on $F^{2}$ (SHELXTL). The refinement converged to $R_{1}=0.033[F>4 \sigma(F)], w R_{2}$ $=0.136$ (all data) and $G O F=0.890$. Absolute structure parameter $=0.05(6)$.

Compound $(\boldsymbol{S}, \boldsymbol{S})-\mathbf{5} . \mathrm{C}_{24} \mathrm{H}_{23} \mathrm{N0}_{5} \mathrm{~S}, M=437.49$, triclinic space group $P 1, a=8.056(5) \AA$, $b=8.326(5) \AA, c=8.751(6) \AA, \alpha=77.78(5)^{\circ}, \beta=78.13(5)^{\circ}, \gamma=89.88(5)^{\circ}, V=$ $560.9(6) \AA^{3}, Z=1, D c=1.295 \mathrm{~g} \mathrm{~cm}^{-3}, \lambda($ Mo K $\alpha)=0.71073 \AA, T=293(2), \mu($ Mo K $\alpha)=$ $0.179 \mathrm{~mm}^{-1}$. A well-shaped crystal of dimensions $0.18 \times 0.19 \times 0.24 \mathrm{~mm}$ was mounted on a Siemens P4 diffractometer. A total of 8553 independent reflections were collected. The structure was solved by direct methods and refined by full-matrix least-squares analysis on $F^{2}$ (SHELXTL). The refinement converged to $R_{1}=0.049[F>4 \sigma(F)], w R_{2}$ $=0.132$ (all data) and $G O F=1.117$. Absolute structure parameter $=0.02(11)$.

Compound $(\boldsymbol{R}, \boldsymbol{S})-2 . \mathrm{C}_{26} \mathrm{H}_{24} \mathrm{~N}_{2} \mathrm{O}_{4} \mathrm{~S}, M=460.53$, monoclinic space group $P 2{ }_{1}, a=$ 5.0372(12) $\AA, b=23.824(5) \AA, c=9.899(2) \AA, \beta=98.69(2)^{\circ}, V=1174.3(5) \AA^{3}, Z=2$, $D c=1.302 \mathrm{~g} \mathrm{~cm}^{-3}, \lambda($ Mo K $\alpha)=0.71073 \AA, T=293(2), \mu($ Mo K $\alpha)=0.173 \mathrm{~mm}^{-1}$. A well-shaped crystal of dimensions $0.17 \times 0.18 \times 0.20 \mathrm{~mm}$ was mounted on a Siemens P4 diffractometer. A total of 8553 independent reflections were collected. The structure was solved by direct methods and refined by full-matrix least-squares analysis on $F^{2}$ (SHELXTL). The refinement converged to $R_{1}=0.044[F>4 \sigma(F)], w R_{2}=0.133$ (all data) and $G O F=0.900$. Absolute structure parameter $=0.0(2)$. 
Table 1. Selected bond lengths $[\AA]$ and angles $\left[{ }^{\circ}\right]$ for $(\boldsymbol{R}, S)-2$.

\begin{tabular}{llll}
\hline $\mathrm{O}(1)-\mathrm{C}(5)$ & $1.232(5)$ & $\mathrm{C}(4)-\mathrm{C}(5)$ & $1.446(6)$ \\
$\mathrm{C}(5)-\mathrm{C}(6)$ & $1.493(6)$ & $\mathrm{C}(9)-\mathrm{C}(12)$ & $1.536(6)$ \\
$\mathrm{C}(12)-\mathrm{C}(13)$ & $1.518(6)$ & $\mathrm{C}(12)-\mathrm{C}(14)$ & $1.528(5)$ \\
$\mathrm{O}(2)-\mathrm{C}(14)$ & $1.222(4)$ & $\mathrm{N}(1)-\mathrm{C}(14)$ & $1.335(5)$ \\
$\mathrm{N}(1)-\mathrm{C}(15)$ & $1.446(5)$ & $\mathrm{C}(15)-\mathrm{C}(16)$ & $1.512(6)$ \\
$\mathrm{C}(15)-\mathrm{C}(18)$ & $1.539(6)$ & $\mathrm{C}(18)-\mathrm{C}(19)$ & $1.478(6)$ \\
& & & \\
$\mathrm{O}(1)-\mathrm{C}(5)-\mathrm{C}(4)$ & $120.7(4)$ & $\mathrm{O}(1)-\mathrm{C}(5)-\mathrm{C}(6)$ & $119.6(4)$ \\
$\mathrm{C}(4)-\mathrm{C}(5)-\mathrm{C}(6)$ & $119.7(4)$ & $\mathrm{C}(13)-\mathrm{C}(12)-\mathrm{C}(14)$ & $111.8(3)$ \\
$\mathrm{C}(13)-\mathrm{C}(12)-\mathrm{C}(9)$ & $115.6(4)$ & $\mathrm{C}(14)-\mathrm{C}(12)-\mathrm{C}(9)$ & $104.2(3)$ \\
$\mathrm{O}(2)-\mathrm{C}(14)-\mathrm{N}(1)$ & $122.0(3)$ & $\mathrm{O}(2)-\mathrm{C}(14)-\mathrm{C}(12)$ & $121.4(4)$ \\
$\mathrm{N}(1)-\mathrm{C}(14)-\mathrm{C}(12)$ & $116.5(3)$ & $\mathrm{C}(14)-\mathrm{N}(1)-\mathrm{C}(15)$ & $122.3(3)$ \\
$\mathrm{N}(1)-\mathrm{C}(15)-\mathrm{C}(16)$ & $111.6(3)$ & $\mathrm{N}(1)-\mathrm{C}(15)-\mathrm{C}(18)$ & $110.3(3)$ \\
$\mathrm{C}(16)-\mathrm{C}(15)-\mathrm{C}(18)$ & $110.9(3)$ & $\mathrm{C}(19)-\mathrm{C}(18)-\mathrm{C}(15)$ & $112.9(3)$ \\
& & & \\
\hline
\end{tabular}

Table 2. Selected bond lengths $[\AA]$ and angles $\left[{ }^{\circ}\right]$ for $(\boldsymbol{S}, \boldsymbol{S})-\mathbf{3}$.

\begin{tabular}{llll}
\hline $\mathrm{O}(1)-\mathrm{C}(7)$ & $1.223(3)$ & $\mathrm{C}(6)-\mathrm{C}(7)$ & $1.489(4)$ \\
$\mathrm{C}(7)-\mathrm{C}(8)$ & $1.449(4)$ & $\mathrm{C}(11)-\mathrm{C}(12)$ & $1.514(4)$ \\
$\mathrm{C}(12)-\mathrm{C}(13)$ & $1.524(4)$ & $\mathrm{C}(12)-\mathrm{C}(14)$ & $1.510(4)$ \\
$\mathrm{O}(2)-\mathrm{C}(14)$ & $1.238(3)$ & $\mathrm{N}(1)-\mathrm{C}(14)$ & $1.333(3)$ \\
$\mathrm{N}(1)-\mathrm{C}(15)$ & $1.439(4)$ & $\mathrm{C}(15)-\mathrm{C}(16)$ & $1.512(4)$ \\
$\mathrm{C}(15)-\mathrm{C}(18)$ & $1.535(4)$ & $\mathrm{C}(18)-\mathrm{C}(19)$ & $1.508(4)$ \\
& & & \\
$\mathrm{O}(1)-\mathrm{C}(7)-\mathrm{C}(8)$ & $120.0(3)$ & $\mathrm{O}(1)-\mathrm{C}(7)-\mathrm{C}(6)$ & $119.2(3)$ \\
$\mathrm{C}(8)-\mathrm{C}(7)-\mathrm{C}(6)$ & $120.8(2)$ & $\mathrm{C}(14)-\mathrm{C}(12)-\mathrm{C}(11)$ & $107.6(2)$ \\
$\mathrm{C}(14)-\mathrm{C}(12)-\mathrm{C}(13)$ & $111.5(2)$ & $\mathrm{C}(11)-\mathrm{C}(12)-\mathrm{C}(13)$ & $112.7(2)$ \\
$\mathrm{O}(2)-\mathrm{C}(14)-\mathrm{N}(1)$ & $121.9(2)$ & $\mathrm{O}(2)-\mathrm{C}(14)-\mathrm{C}(12)$ & $121.4(2)$ \\
$\mathrm{N}(1)-\mathrm{C}(14)-\mathrm{C}(12)$ & $116.6(2)$ & $\mathrm{C}(14)-\mathrm{N}(1)-\mathrm{C}(15)$ & $123.7(2)$ \\
$\mathrm{N}(1)-\mathrm{C}(15)-\mathrm{C}(16)$ & $113.3(2)$ & $\mathrm{N}(1)-\mathrm{C}(15)-\mathrm{C}(18)$ & $110.4(2)$ \\
$\mathrm{C}(16)-\mathrm{C}(15)-\mathrm{C}(18)$ & $109.0(2)$ & $\mathrm{C}(19)-\mathrm{C}(18)-\mathrm{C}(15)$ & $114.0(2)$ \\
& & & \\
\hline
\end{tabular}


Table 3. Selected bond lengths $[\AA]$ and angles $\left[{ }^{\circ}\right]$ for $(\boldsymbol{S}, \boldsymbol{S})-\mathbf{5}$.

\begin{tabular}{llll}
\hline $\mathrm{O}(1)-\mathrm{C}(5)$ & $1.224(5)$ & $\mathrm{C}(4)-\mathrm{C}(5)$ & $1.454(6)$ \\
$\mathrm{C}(5)-\mathrm{C}(6)$ & $1.502(6)$ & $\mathrm{C}(9)-\mathrm{C}(12)$ & $1.528(6)$ \\
$\mathrm{C}(12)-\mathrm{C}(13)$ & $1.539(7)$ & $\mathrm{C}(12)-\mathrm{C}(14)$ & $1.543(6)$ \\
$\mathrm{O}(2)-\mathrm{C}(14)$ & $1.209(5)$ & $\mathrm{N}(1)-\mathrm{C}(14)$ & $1.334(5)$ \\
$\mathrm{N}(1)-\mathrm{C}(15)$ & $1.454(5)$ & $\mathrm{C}(15)-\mathrm{C}(16)$ & $1.534(6)$ \\
$\mathrm{C}(15)-\mathrm{C}(18)$ & $1.520(7)$ & $\mathrm{C}(18)-\mathrm{C}(19)$ & $1.516(6)$ \\
& & & \\
$\mathrm{O}(1)-\mathrm{C}(5)-\mathrm{C}(4)$ & $118.5(4)$ & $\mathrm{O}(1)-\mathrm{C}(5)-\mathrm{C}(6)$ & $120.2(4)$ \\
$\mathrm{C}(4)-\mathrm{C}(5)-\mathrm{C}(6)$ & $121.3(4)$ & $\mathrm{C}(9)-\mathrm{C}(12)-\mathrm{C}(13)$ & $113.1(4)$ \\
$\mathrm{C}(9)-\mathrm{C}(12)-\mathrm{C}(14)$ & $107.8(3)$ & $\mathrm{C}(13)-\mathrm{C}(12)-\mathrm{C}(14)$ & $110.1(4)$ \\
$\mathrm{O}(2)-\mathrm{C}(14)-\mathrm{N}(1)$ & $122.5(4)$ & $\mathrm{O}(2)-\mathrm{C}(14)-\mathrm{C}(12)$ & $123.5(4)$ \\
$\mathrm{N}(1)-\mathrm{C}(14)-\mathrm{C}(12)$ & $113.9(4)$ & $\mathrm{C}(14)-\mathrm{N}(1)-\mathrm{C}(15)$ & $122.6(3)$ \\
$\mathrm{N}(1)-\mathrm{C}(15)-\mathrm{C}(18)$ & $114.3(4)$ & $\mathrm{N}(1)-\mathrm{C}(15)-\mathrm{C}(16)$ & $111.7(4)$ \\
$\mathrm{C}(18)-\mathrm{C}(15)-\mathrm{C}(16)$ & $112.8(4)$ & $\mathrm{C}(19)-\mathrm{C}(18)-\mathrm{C}(15)$ & $112.2(4)$ \\
& & & \\
& & &
\end{tabular}

Table 4. Selected bond lengths $[\AA]$ and angles $\left[{ }^{\circ}\right]$ for $(\boldsymbol{R}, \boldsymbol{S})-\mathbf{5}$.

\begin{tabular}{llll}
\hline $\mathrm{O}(1)-\mathrm{C}(5)$ & $1.222(3)$ & $\mathrm{C}(4)-\mathrm{C}(5)$ & $1.466(3)$ \\
$\mathrm{C}(5)-\mathrm{C}(6)$ & $1.496(3)$ & $\mathrm{C}(9)-\mathrm{C}(12)$ & $1.520(3)$ \\
$\mathrm{C}(12)-\mathrm{C}(13)$ & $1.530(4)$ & $\mathrm{C}(12)-\mathrm{C}(14)$ & $1.511(4)$ \\
$\mathrm{O}(2)-\mathrm{C}(14)$ & $1.233(3)$ & $\mathrm{N}(1)-\mathrm{C}(14)$ & $1.334(3)$ \\
$\mathrm{N}(1)-\mathrm{C}(15)$ & $1.449(3)$ & $\mathrm{C}(15)-\mathrm{C}(16)$ & $1.515(3)$ \\
$\mathrm{C}(15)-\mathrm{C}(18)$ & $1.533(4)$ & $\mathrm{C}(18)-\mathrm{C}(19)$ & $1.513(4)$ \\
& & & \\
$\mathrm{O}(1)-\mathrm{C}(5)-\mathrm{C}(4)$ & $118.9(2)$ & $\mathrm{O}(1)-\mathrm{C}(5)-\mathrm{C}(6)$ & $120.5(2)$ \\
$\mathrm{C}(4)-\mathrm{C}(5)-\mathrm{C}(6)$ & $120.6(2)$ & $\mathrm{C}(14)-\mathrm{C}(12)-\mathrm{C}(9)$ & $106.9(2)$ \\
$\mathrm{C}(14)-\mathrm{C}(12)-\mathrm{C}(13)$ & $111.1(2)$ & $\mathrm{C}(9)-\mathrm{C}(12)-\mathrm{C}(13)$ & $113.0(2)$ \\
$\mathrm{O}(2)-\mathrm{C}(14)-\mathrm{N}(1)$ & $121.8(2)$ & $\mathrm{O}(2)-\mathrm{C}(14)-\mathrm{C}(12)$ & $121.4(2)$ \\
$\mathrm{N}(1)-\mathrm{C}(14)-\mathrm{C}(12)$ & $116.6(2)$ & $\mathrm{C}(14)-\mathrm{N}(1)-\mathrm{C}(15)$ & $122.4(2)$ \\
$\mathrm{N}(1)-\mathrm{C}(15)-\mathrm{C}(16)$ & $114.5(2)$ & $\mathrm{N}(1)-\mathrm{C}(15)-\mathrm{C}(18)$ & $109.8(2)$ \\
$\mathrm{C}(16)-\mathrm{C}(15)-\mathrm{C}(18)$ & $109.4(2)$ & $\mathrm{C}(19)-\mathrm{C}(18)-\mathrm{C}(15)$ & $114.1(2)$ \\
& & & \\
\hline
\end{tabular}




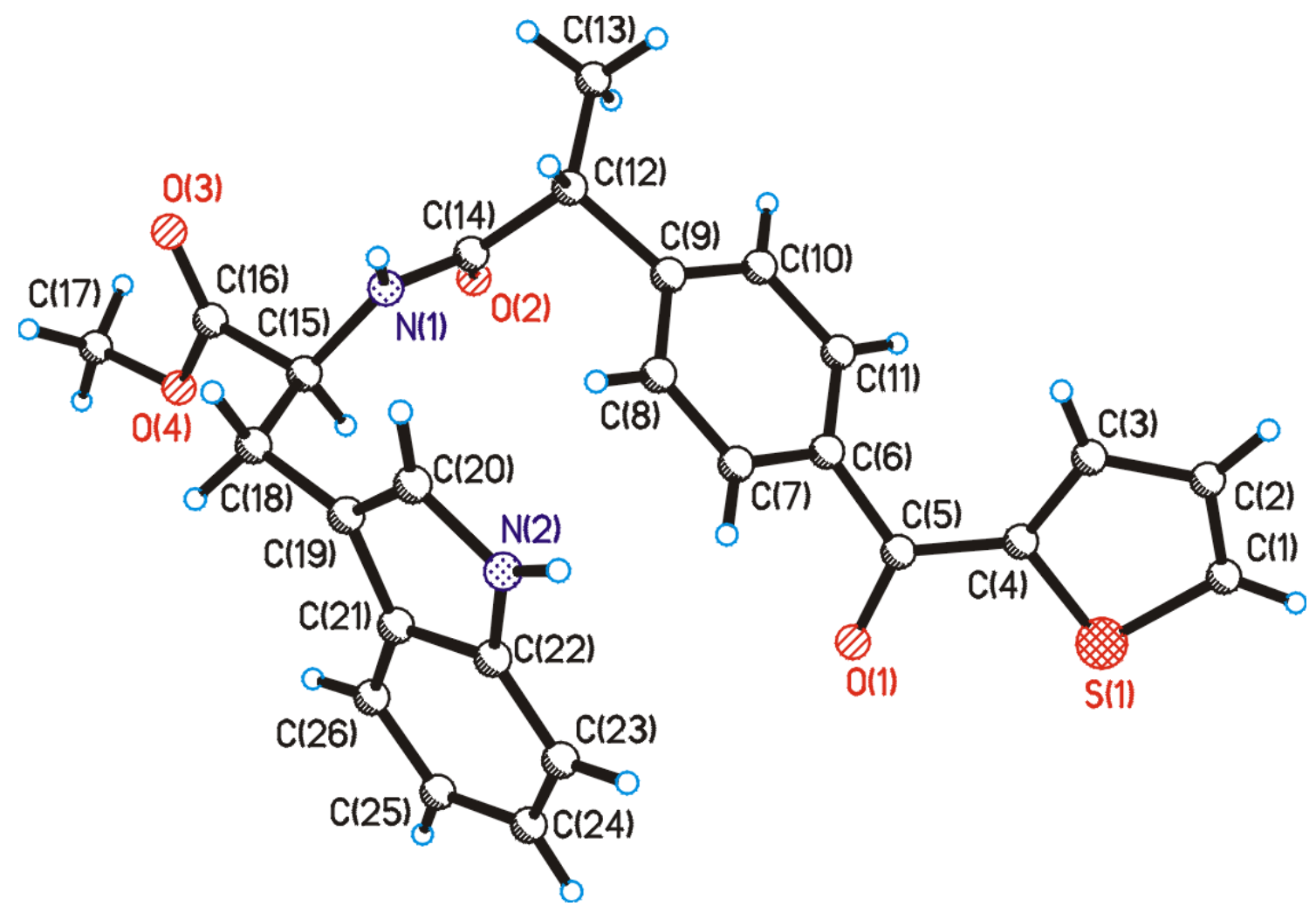

Molecular structure of $(\boldsymbol{R}, S)-2$ 


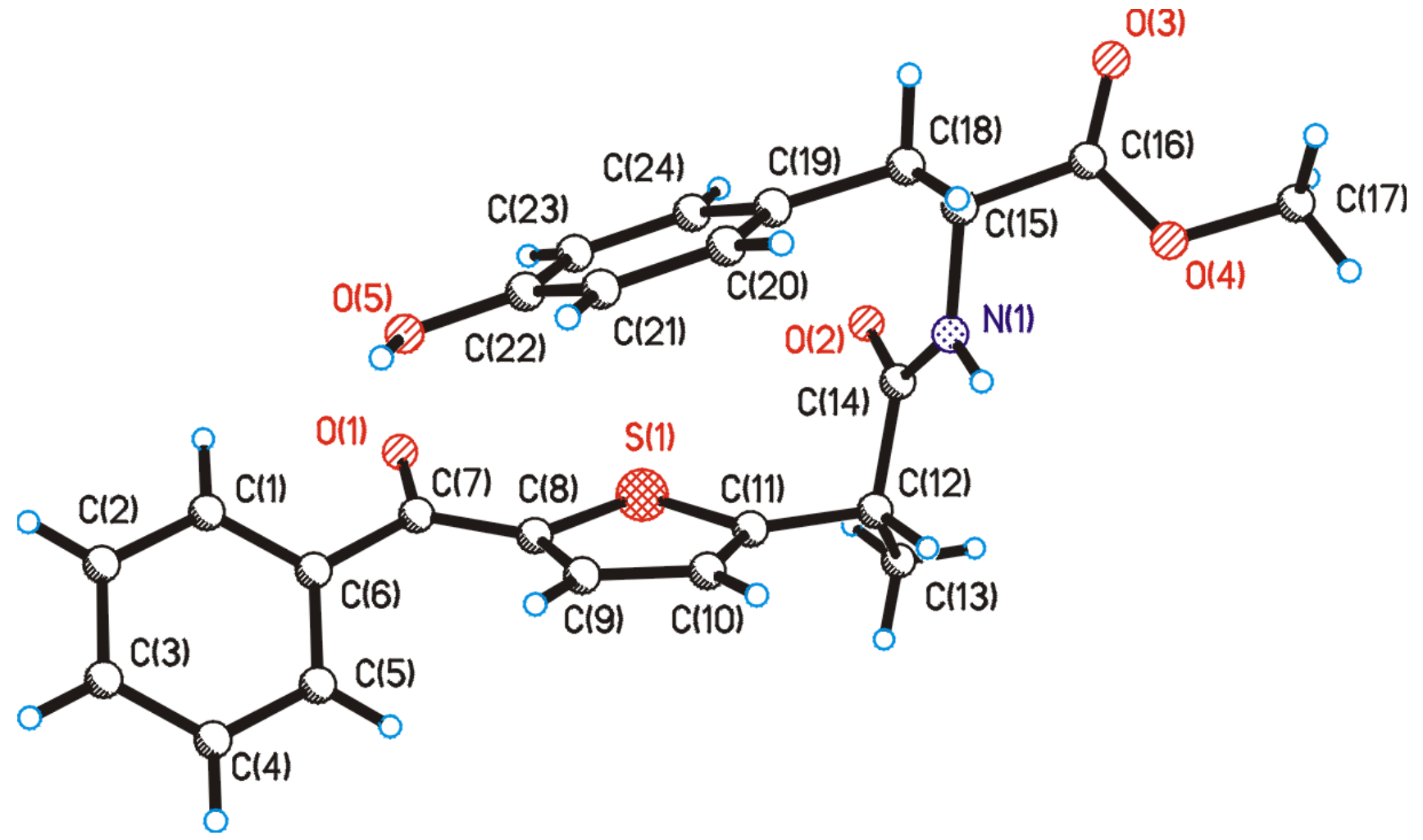

Molecular structure of $(S, S)-3$ 


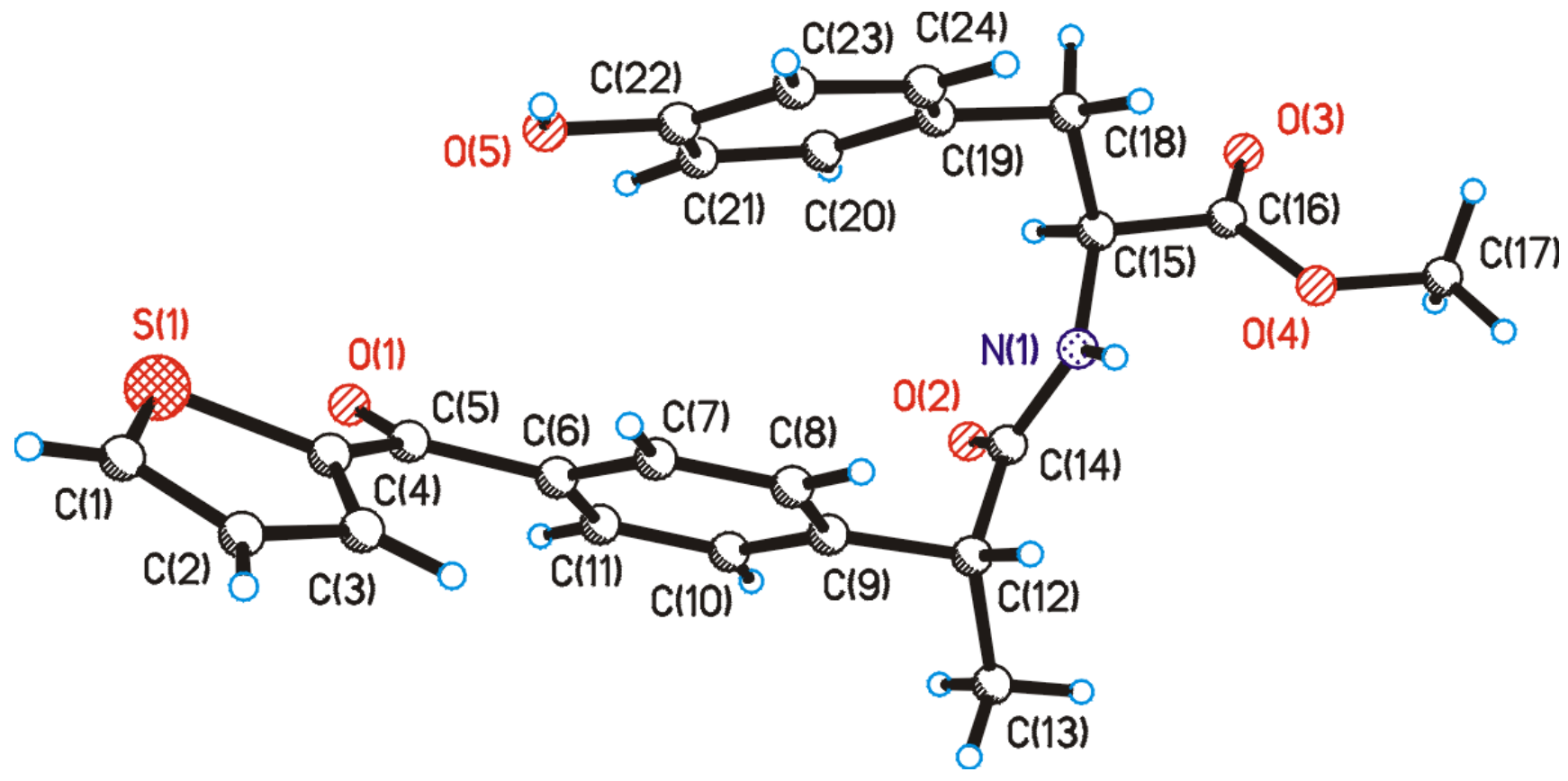

Molecular structure of $(\boldsymbol{R}, S)-5$ 


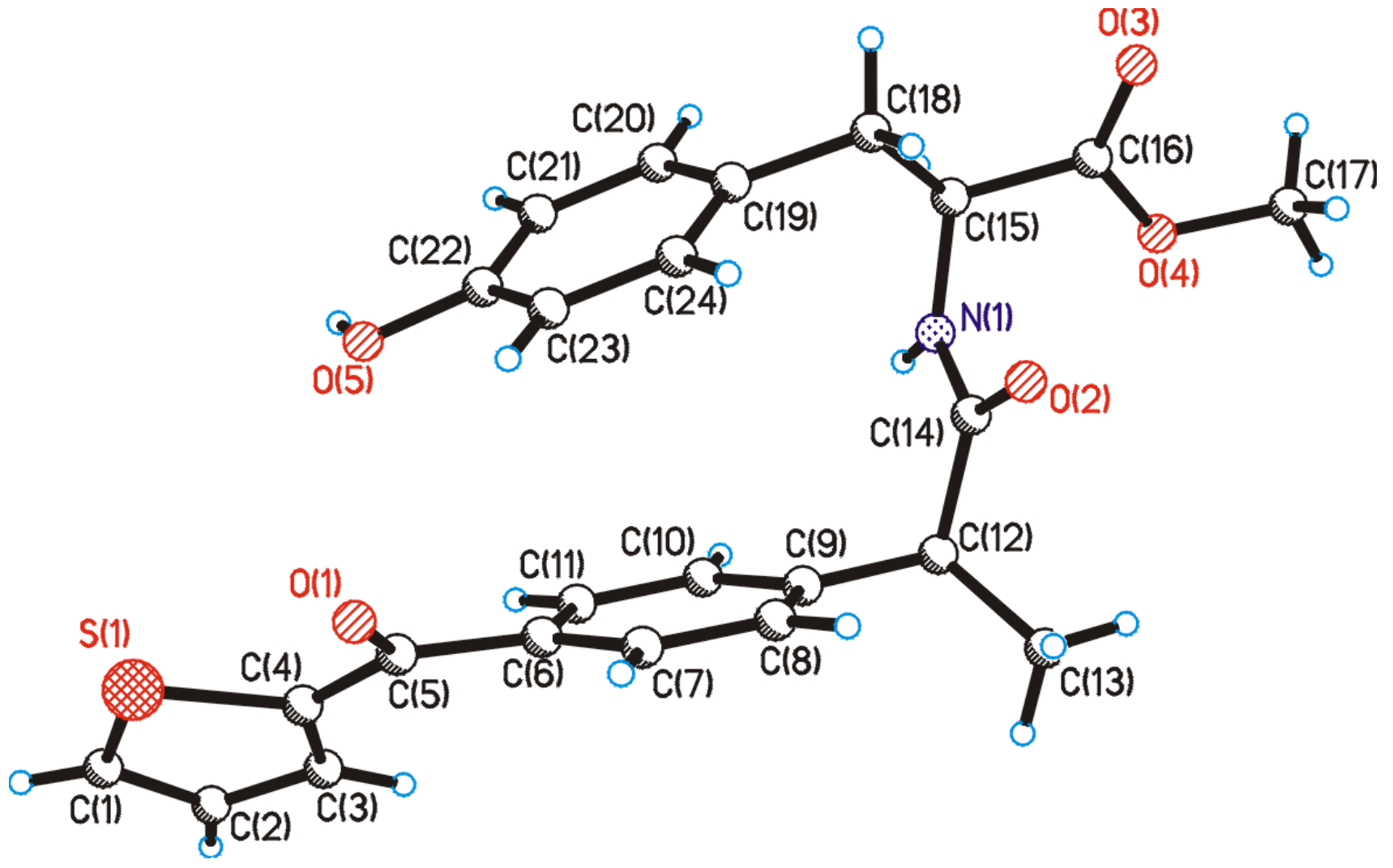

Molecular structure of $(\boldsymbol{S}, S)-5$ 